\title{
Características de la avifauna en un fragmento de bosque húmedo premontano afectado por ruido vehicular
}

\section{Jessica Nathalia Sánchez-Guzmán, ${ }^{*}$ Sergio Losada-Prado}

Universidad del Tolima, Facultad de Ciencias, Programa de Biología, Grupo de Investigación en Zoología. Apartado Aéreo 546, Barrio Santa Helena Parte Alta, Ibagué, Colombia.

*Autor para correspondencia: jessicanathaliasanchez@hotmail.com

\section{RESUMEN}

La contaminación acústica producida por las carreteras representa uno de los factores que afecta en mayor medida la presencia, densidad y diversidad de la avifauna. En este estudio se evaluó la asociación entre el ruido vehicular, el número de especies de aves canoras y el número de detecciones en un fragmento de bosque ripario con alta influencia urbana. Se realizaron muestreos mensuales entre septiembre y noviembre de 2014 de 6:00 a 9:00 a. m. en un fragmento de bosque húmedo premontano dentro de la Universidad del Tolima. Las aves vocalizadoras se grabaron durante diez $\mathrm{min} /$ hora y se registró el valor máximo de ruido (dB) obtenido en dos minutos. Los cantos se analizaron empleando Audacity ${ }^{\circledR}$ y la determinación de las especies se realizó consultando a expertos y confrontando con bases de datos. Se identificaron cantos de 43 especies principalmente de la familia Thraupidae y Tyrannidae. Se registraron diferencias significativas en el número de especies $\left(\mathrm{F}_{3,47}=4,38 ; p=0,025\right)$ y el número de detecciones $\left(\mathrm{F}_{3,47}=4,51 ; p=0,02\right)$ entre meses, y en el número de especies $\left(\mathrm{F}_{3,1}=3,14 ; p=0,05\right)$ y el número de detecciones $\left(\mathrm{F}_{3,1}=6,03 ; p=0,004\right)$ entre horas. No hubo diferencias significativas en las variables en relación a la intensidad del ruido vehicular, ni en los valores de ruido en los meses y horas muestreadas. Finalmente, se evidenció un aumento en el número de detecciones a medida que se genera la transición del período seco al lluvioso, por lo cual se recomienda realizar estudios de este tipo a escala temporal anual.

Palabras claves: bioacústica, cantos, detecciones, frecuencia, vocalizaciones.

Editor: Hernández Fernández, J.

javier.hernandez@utadeo.edu.co

Citation: Sánchez-Guzmán, J. N. \& Losada-Prado, S. (2016). Características de la avifauna en un fragmento de bosque húmedo premontano afectado por ruido vehicular. Mutis 6(2), 7-18, doi: http://dx.doi. org/10.21789/22561498.1147

Received: Marzo 10, 2016. Accepted: Junio 15, 2016. Published on line: Septiembre 30, 2016.

Copyright: $\odot 2016$ Sánchez-Guzmán, J. N. \& Losada-Prado, S. This is an open-access article, which permits unrestricted use, distributions and reproduction in any me $\neg$ dium, provided the original author and source are credited.

Competing Interests: The authors have no conflict of interest.
Features of the avifauna in a fragment of premontane moist forest affected by vehicular noise

\begin{abstract}
The noise pollution caused by road is one of the factors that more affect the presence, density and diversity of birds. In this study was evaluated the association between vehicular noise and, the number of species of songbirds and detections on a fragment of riparian forest with high urban influence.
\end{abstract}


Monthly samplings were made between september and november of 2014 from 06:00 to 09:00 h on a fragment of premontane moist forest within the University of Tolima. The song birds were recorded for ten $\mathrm{min} /$ hour and the maximum value of noise $(\mathrm{dB})$ obtained in two minutes was recorded. The songs were analyzed using Audacity ${ }^{\circledR}$ and determination of species was conducted by consulting experts and confronting with databases. Were identified songs of 43 species mainly from the family Tyrannidae and Thraupidae. Significant differences were registered in the number of species $\left(\mathrm{F}_{3,47}=4.38 ; p=0.025\right)$ and the number of detections $\left(\mathrm{F}_{3,47}=4.51 ; p=0.02\right)$ between months, and the number of species $\left(\mathrm{F}_{3.1}=3.14 ; p=\right.$ $0.05)$ and the number of detections $\left(\mathrm{F}_{3.1}=6.03 ; p=\right.$ $0.004)$ between hours. There were no significant differences in the variables in relation to the intensity of vehicular noise, or noise values in the months and hours sampled. Finally, an increase in the number of detections as the transition from dry to rainy season was evident generated, so it is recommended that realize such studies to annual time scale.

Keywords: bioacoustic, detections, frequency, songs, vocalizations.

\section{INTRODUCCIÓN}

La contaminación acústica derivada del ruido vehicular ha sido reconocida desde hace décadas como un importante problema ambiental (Ruiz, Rubines y Lahoz, 2006). En la actualidad dicho factor se ha extendido en el tiempo y en el espacio debido principalmente al dinámico desarrollo de la infraestructura urbana y vial (Fahrig, Pedla, Pope, Taylor y Wegner, 1995; McGregor, Bender y Fahrig, 2008; Pisanty et al., 2009), constituyendo una amenaza para las poblaciones silvestres. Entre los grupos taxonómicos más afectados se encuentran los anfibios, en los cuales se ha evidenciado que un disturbio sensorial externo como el ruido de los vehículos puede afectar el proceso de hibernación (Brattstrom y BondeIlo, 1983), alterar la comunicación durante la época de cortejo y cría (Wollerman y Willey, 2002; Bee y Swanson, 2007; Lengagne, 2008; Parris, Velik-Lord y North, 2009), generar cambios en la actividad locomotora (Lukanov, Simeonovska-Nikolova y Tzankov, 2014), e inhibir o promover la actividad vocal en algunas especies (Sun y Narins, 2005), entre otras consecuencias. Por otro lado, en los mamíferos se ha do- cumentado que en especies sensibles a la presencia humana el ruido vehicular provoca abandono o no selección de áreas con influencia sonora de tráfico (Rost y Bailey, 1979; Mace, Waller, Manley, Lyon y Zuuring, 1996).

Por su parte, las aves debido a su recepción de sonidos para su comunicación intra e interespecífica y demás actividades cotidianas (Ruiz et al., 2006), constituyen una las clases más afectadas por este problema, reportándose que el ruido antropogénico puede enmascarar los efectos acústicos, disminuyendo la eficacia de los llamados de alerta, señales de defensa territorial y apareamiento (Slabbekoorn y Peet, 2003; Slabbekoorn y den Boer-Visser, 2006), lo cual trae consecuencias demográficas graves como cambios en la abundancia y en la estructura de la comunidad (Francis, Ortega y Cruz, 2009). Se han realizado estudios que han evidenciado el efecto del ruido antropogénico en los cantos de las aves, los cuales han sido desarrollados principalmente en el hemisferio norte y han evaluado aspectos como cambios en las características del canto (Francis, Ortega y Cruz, 2011; Slabbekoorn, Yang y Halfwerk, 2012), en los patrones de ocupación (Goodwin y Shriver, 2013), y en la densidad poblacional y de cría (Peris y Pescador, 2004; Arévalo y Newhard, 2011), entre otros.

Entre los estudios realizados en Latinoamérica (Mendes, Colino-Rabanal y Peris, 2011; Mendoza y Arce-Plata, 2012; Pacheco-Vargas y Losada-Prado, 2015), son pocos los que evalúan específicamente el efecto del ruido vehicular en ambientes urbanos y sus consecuencias sobre las características de la comunidad. Tal es el caso de Perepelizin y Faggi (2009), quienes analizaron cómo las diferencias en la trama urbana condicionan la presencia y densidad de aves; dichos autores concluyen que una mayor presencia de aves se asocia directamente a una menor densidad poblacional humana y niveles de actividad y ruido menores. Por otro lado, Llavallol, Cellini y Gutiérrez (2012) registraron que no hay relación entre la variación del comportamiento, riqueza y abundancia de aves en el bosque de arrayanes (península de Quetrihue, Argentina) y la generación de ruidos en general. El objetivo de este estudio fue evaluar la asociación entre el ruido vehicular constante en diferentes meses y horas de la mañana, sobre el número de detecciones y de especies de aves canoras en un fragmento de bosque ripario con alta influencia urbana. 


\section{MATERIALES Y MÉTODOS}

\section{Área de estudio}

El fragmento de bosque de este estudio se localiza en predios del Jardín Botánico Alejandro von Humboldt de la Universidad del Tolima, al sur del municipio de Ibagué, a $4^{\circ} 25^{\prime} 31^{\prime \prime} \mathrm{N}$ y $75^{\circ} 12^{\prime} 30^{\prime \prime} \mathrm{W}$, en altitudes que oscilan entre 1070 y $1170 \mathrm{msnm}$. Su temperatura promedio es de $23^{\circ} \mathrm{C}$ (entre 22,5 y $26,5^{\circ} \mathrm{C}$ ) y presenta una precipitación anual de $1193 \mathrm{~mm}$ (Moreno-Palacios, Jeyson-Sanabria, Díaz-Jaramillo, Carantón-Ayala y Parra-Hernández, 2007). Cuenta con una extensión de diez hectáreas (ha), de las cuales el $40 \%$ es terreno plano y el restante $60 \%$ pendiente. Presenta una vegetación típica de bosque seco tropical, bosque húmedo subandino y bosque húmedo andino, que sustenta aproximadamente 516 especies vegetales (exóticas y nativas) y sirve de hábitat para muchas especies animales (Moreno-Palacios et al., 2007; Ospina-López y Reinoso-Flórez, 2009). Además, limita al sur a una distancia promedio de aproximadamente $75 \mathrm{~m}$ con la vía Panamericana (Variante), la cual comunica a Armenia con Bogotá, y se encuentra continuamente transitada por toda clase de vehículos, siendo fuente constante de ruido.

\section{Modelo de estudio}

En la valoración de la calidad de hábitats urbanos es usual emplear a las aves como indicadoras, por ser un grupo muy diversificado ecológica y taxonómicamente, de distribución mundial y con una marcada sensibilidad a los cambios ambientales (Fernández-Juricic, 2000). Para este estudio, se seleccionó esta clase como indicador pues dentro de ella se hallan especies con comportamientos y sensibilidad variada ante el ruido (Goosem, 2002; Llavallol, et al., 2012).

\section{Colecta de datos}

Se llevaron a cabo dos muestreos mensuales en septiembre, octubre y noviembre de 2014. La grabación de los cantos se realizó durante diez minutos a las 6:00, 7:00, 8:00 y 9:00 horas dentro del área de estudio en tres puntos separados uno del otro por una distancia mínima de $\mathbf{3 0}$ metros (los cuales fueron seleccionados a partir de un premuestreo realizado en el mes de agosto), empleando un equipo de bioacústica compuesto por una grabadora digital Marantz PMD
661, un micrófono unidireccional Sennheiser ME62 y una antena parabólica Sennheiser. Para la medición del ruido (decibeles, dB) se empleó un sonómetro Extech 407730 y se realizó durante el registro de las grabaciones, obteniéndose su valor mediante el promedio de dos mediciones de dos minutos por cada hora de estudio, las cuales se realizaron en ausencia de lluvia o viento fuerte.

La revisión de los cantos se hizo empleando el software de edición de audio Audacity ${ }^{\circledR}$ versión 2.0.5. (Audacity Team, 2013) (formato de muestra 32 bits flotante; frecuencia real $44100 \mathrm{~Hz}$; velocidad 1,00 X), con el fin de visualizar el espectrograma, escuchar e identificar la especie que está vocalizando y hacer los recortes de cada grabación para posteriormente ingresar aquellas de mejor calidad a la colección bioacústica de la Universidad del Tolima (CBUT). El proceso de determinación de la especie vocalizadora se llevó a cabo confrontando las grabaciones y recortes en la base de datos Xeno-canto, con las grabaciones almacenadas en la CBUT y consultando a expertos.

\section{Análisis de datos}

Las características de la comunidad de aves en cada hora se expresaron como la frecuencia de ocurrencia absoluta (FOA: número de muestreos en los que cada especie se registró) y relativa (FOR: [número de muestreos en que cada especie se registró/total de muestreos] ${ }^{*} 100$ ) de las especies detectadas; la abundancia relativa ([frecuencia de ocurrencia absoluta/total individuos detectados]*100); y el número de especies detectadas en una sola hora de la mañana o con una sola detección durante todo el estudio. Además, se empleó un índice de importancia relativa (IIR), modificado a partir del índice utilizado por Bucher y Herrera (1981), como estimador general de la importancia de cada especie en el área de estudio (Gatto, Quintana, Yorio y Lisnizer, 2005), según la expresión IIR = $100 \mathrm{pi}(\mathrm{Si}+\mathrm{Ei}) /(\mathrm{St}+\mathrm{Et})$, donde $P i=$ ni/N (ni: número total de detecciones de la especie; $N$ : número total de la suma de todas las detecciones de todas las especies), Si es el número de días en los cuales la especie $i$ estuvo presente, Ei es el número de horas en las cuales la especie $i$ estuvo presente, y St y Et el número total de días y horas de muestreo, respectivamente.

Los efectos de la hora, el mes y la intensidad de ruido vehicular sobre el número de especies y el número de 
detecciones se determinaron aplicando análisis de varianza (ANOVA), previa confirmación del cumplimiento de los supuestos de normalidad (Shapiro-Wilks) y homogeneidad de varianzas (prueba F) (Di Rienzo et al., 2013). En el caso de la relación entre la intensidad del ruido vehicular y las horas y meses muestreados, los supuestos mencionados fueron infringidos, por tal se aplicó el método no paramétrico de Kruskall-Wallis. Todos los análisis estadísticos se realizaron mediante el programa InfoStat ${ }^{\circledR}$ (Di Rienzo et al., 2013).

\section{RESULTADOS}

\section{Características de la comunidad}

A través de registros bioacústicos se reconocieron 43 especies de aves, distribuidas en 11 órdenes y 19 familias. La familia más numerosa fue Thraupidae con 13 especies, seguida de Tyrannidae con siete especies y Troglodytidae con tres especies (Anexo 1). En cuanto al número de detecciones, la familia con más registros fue Thraupidae con 65 detecciones, seguida por Vireonidae, Troglodytidae y Psittacidae con 48,42 y 37 detecciones respectivamente. Las especies más vocalizadoras fueron Brotogeris jugularis y Troglodytes aedon con 37 detecciones, seguidas por Myiothlypis fulvicauda con 31, constituyendo dichas especies el $31,44 \%$ de los registros totales.

Se registraron seis especies durante todos los muestreos (FOR del 100\%): M. fulvicauda, Pitangus sulphuratus, Ramphocelus dimidiatus, Saltator striatipectus, Thraupis episcopus y T. aedon (Anexo 1), las cuales presentaron la abundancia relativa más alta con un valor de 2,10. Para el índice de importancia relativa, T. aedon obtuvo el valor superior seguido por $B$. jugularis y M. fulvicauda, lo cual indica que estas especies fueron las más detectadas durante mayor número de días y horas de muestreo. Veinte de las especies registradas $(46,51 \%)$ presentaron un IIR superior a 0,5 (Anexo 1).

Además, se registraron ocho especies durante todos los meses (las seis con FOR del $100 \%$ junto a Hylophilus flavipes y Turdus ignobilis); diez especies en tres meses, ocho especies en dos meses y 17 especies en un solo mes, encontrándose discrepancias en el número de especies en relación al mes $\left(F_{3,47}=4,38\right.$; $p=0,025)$, siendo noviembre y octubre los meses que mostraron mayor número de estas (figura 1). De las especies registradas en un solo mes, Henicorhina leucosticta, Phaeomyias murina, Thraupis palmarum y Todirostrum cinereum se encontraron únicamente en agosto, mes de premuestreo. De igual modo, se encontraron diferencias en el número de detecciones entre meses $\left(\mathrm{F}_{3,47}=4,51 ; p=0,02\right)$, así el mes con mayor número de ellas fue noviembre, debido a que durante las 6:00, 8:00 y 9:00 h presentó mayor registro de individuos vocalizadores; seguidamente se encuentra octubre, mes durante el cual se registró la mayor actividad canora a las 7:00 h (figura 2).

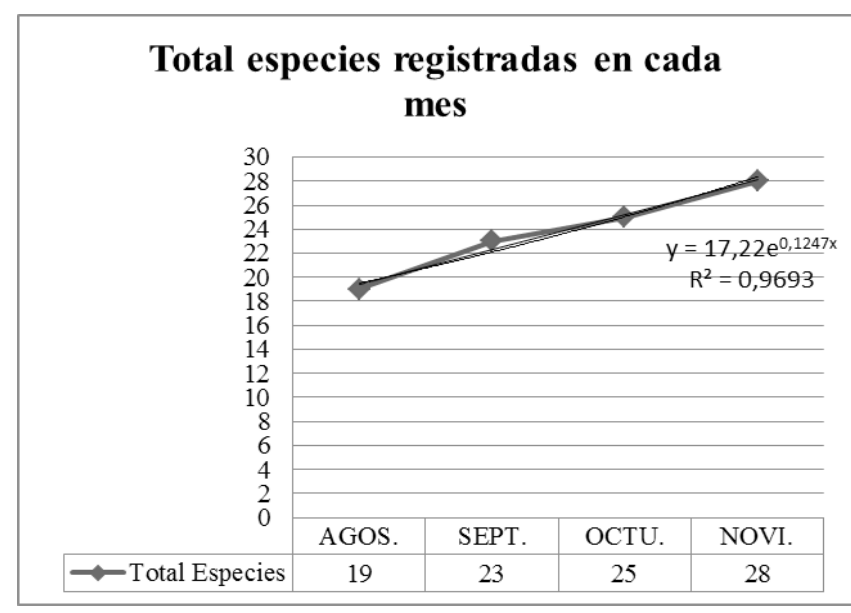

Figura 1. Número de especies registradas bioacústicamente en cada mes de muestreo y premuestreo en el Jardín Botánico Alejandro von Humboldt (agosto-noviembre 2014)

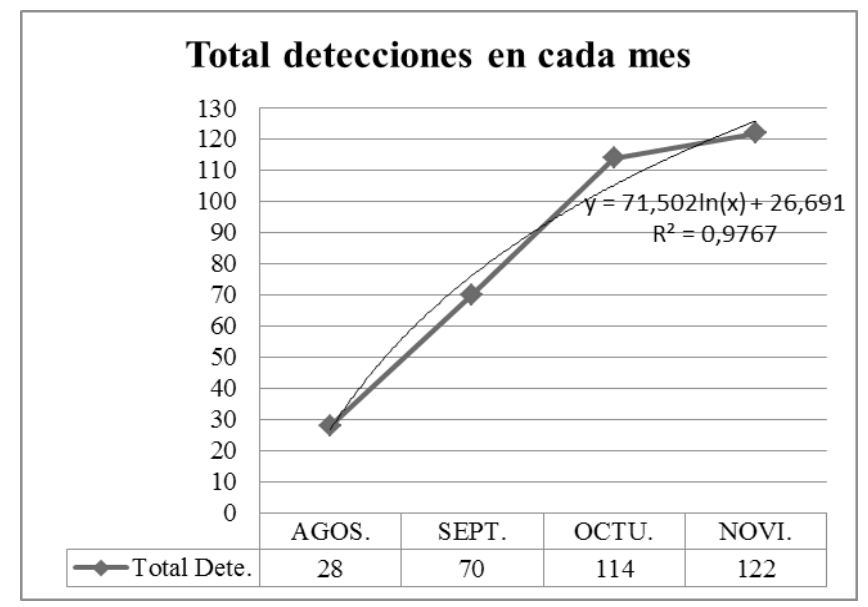

Figura 2. Detecciones totales en cada mes de muestreo y premuestreo en el Jardín Botánico Alejandro von Humboldt (agosto-noviembre 2014) 
Al analizar las vocalizaciones por horas, observamos que 11 especies estuvieron activas durante todas ellas y por tal presentaron una FOR del $100 \%$, siete se registraron en dos y tres horas, y 14 especies vocalizaron exclusivamente en una hora (tabla 1). Adicionalmente, durante el estudio solo a las 6:00 h se detectaron cinco especies; a las 7:00 h, cuatro; a las 8:00 h, dos; y finalmente solo a las 9:00 h, tres. Estas especies se registraron una única vez en todo el muestreo, exceptuando $T$. leucomelas la cual fue reconocida en dos ocasiones a las 6:00 h (tabla 1).

Tabla 1. Especies de aves detectadas en las diferentes horas de muestreo (6:00-9:00 h) en el Jardín Botánico Alejandro von Humboldt

\section{Especies registradas}

\begin{tabular}{|c|c|c|c|c|c|c|}
\hline \multirow[b]{2}{*}{$\begin{array}{c}\text { Todas } \\
\text { las horas }\end{array}$} & \multirow[b]{2}{*}{ Tres horas } & \multirow[b]{2}{*}{ Dos horas } & \multicolumn{4}{|c|}{ Una hora } \\
\hline & & & $6: 00$ & 7:00 & $8: 00$ & 9:00 \\
\hline Brotogeris jugularis & Tachyphonus /uctuosus & Myiozetetes similis & Momotus aequatorialis & Aramides cajaneus & $\begin{array}{c}\text { Basilleuterus } \\
\text { rufifrons }\end{array}$ & Amazilia tzacalt \\
\hline Cyclarhis gujanensis & $\begin{array}{l}\text { Pheugopedius } \\
\text { fasciatoventri }\end{array}$ & Tangara vitriolina & Myrmeciza longipes & Coereba flaveola & Sporophila minuta & Dacnis cayana \\
\hline Hylophilus flavipes & Sicalis flaveola & Tangara cyanicollis & Nyctidromus albicollis & Elaenia chiriquensis & & Elaenia flavogaster \\
\hline Myiothlypis fulvicauda & Columbina talpacoti & Thamnophilus doliatus & Tangara girola & Eucometis penicillata & & \\
\hline Pitangus sulphuratus & Megarynchus pitangua & Vanellus chilensis & Turdus leucomelas & & & \\
\hline Ramphocelus dimidiatus & Malacoptila mystacalis & Euphonia laniirostris & & & & \\
\hline Rupornis magnirostris & Melanerpes rubricapillus & Tiaris bicolor & & & & \\
\hline \multicolumn{7}{|l|}{ Saltator striatipectus } \\
\hline \multicolumn{7}{|l|}{ Troglodytes aedon } \\
\hline \multicolumn{7}{|l|}{ Thraupis episcopus } \\
\hline Turdus ignobilis & & & & & & \\
\hline
\end{tabular}

Por otro lado, las características de la comunidad de aves en las horas evaluadas presentaron cambios notables a medida que transcurría la mañana, de modo tal que existen diferencias en la distribución del número de especies $\left(\mathrm{F}_{3,1}=\right.$ $3,14 ; p=0,05)$, y número de detecciones entre las horas muestreadas $\left(\mathrm{F}_{3,1}=6,03 ; p=0,004\right)$. Así, durante todo el estudio a las 6:00 $\mathrm{h}$ se presentó el mayor número de detecciones y especies; mientras que a las 7:00 y 8:00 h se observó un comportamiento inverso que nos hace formular la posible hipótesis de que las especies que can- tan a la primera hora no lo hacen a la siguiente y viceversa; por su parte a las 9:00 h el número de registros y especies canoras desciende en la comunidad (figura 3). En cuanto a la abundancia, la especie con mayor número de detecciones a las $6: 00$ y $7: 00 \mathrm{~h}$ corresponde a $B$. jugularis con 13 y 12 respectivamente, presentando valores de 4,25 y 3,92; mientras que a las $8: 00$ y 9:00 $\mathrm{h}$ fue $T$. aedon con 10 y 7 detecciones y abundancias de 3,27 y 2,29 junto a $S$. striatipectus con valores iguales en la última hora. 


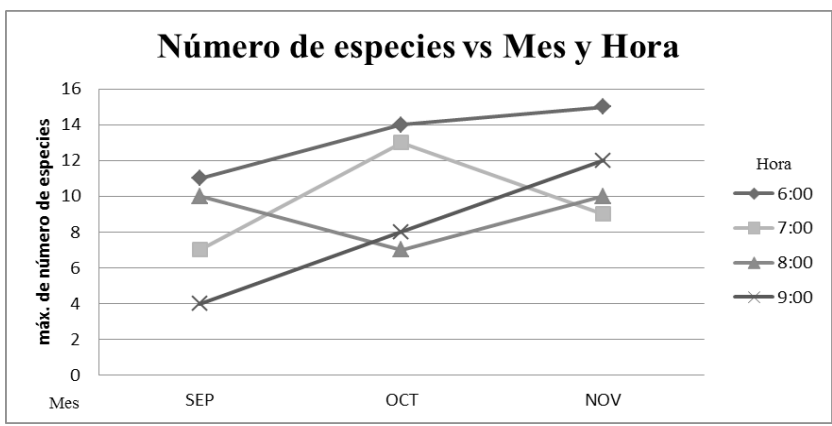

Figura 3. Número máximo de especies por mes y hora a través de registros bioacústicos en el Jardín Botánico Alejandro von Humboldt

\section{Efecto del ruido}

Se registraron valores de ruido de entre $43,9 \mathrm{~dB}$ y $75,7 \mathrm{~dB}$. No se presentaron diferencias en el rui- do vehicular entre meses $(H=1,82 ; p=0,40)$, sin embargo, en promedio el mes que presentó el mayor valor de ruido fue septiembre con 61,36 $\mathrm{dB}$, seguido por octubre con $60,48 \mathrm{~dB}$ y noviembre con 59,75 dB. Tampoco se presentaron diferencias en el ruido vehicular entre horas $(H=3,10$; $p=0,38)$, pero los valores de ruido registrados de mayor a menor siguieron el siguiente orden: 9:00, 8:00, 6:00 y 7:00 h (tabla 2). No se detectaron asociaciones significativas en el número de especies $\left(F_{5}=0,75 ; p=0,41\right)$ o el número de detecciones con relación a la intensidad de ruido vehicular $\left(\mathrm{F}_{5}=0,46 ; p=0,51\right)$; sin embargo, se observó que tanto el número de detecciones como el número de especies decrece al aumentar el promedio de ruido registrado (tabla 2).

Tabla 2. Promedio de ruido registrado en cada mes y hora de la mañana en el Jardín Botánico Alejandro von Humboldt (septiembre-noviembre, 2014)

\begin{tabular}{ccccc}
\hline Mes & Hora & $\mathbf{d B}$ & $\mathbf{N}^{\circ}$ detecciones & $\mathbf{N}^{\circ}$ especies \\
\hline \multirow{3}{*}{ Septiembre } & $6: 00$ & 60,38 & 15 & 10,5 \\
& $7: 00$ & 59,42 & 6 & 5 \\
& $8: 00$ & 59,27 & 10,5 & 8,5 \\
& $9: 00$ & 66,38 & 3,5 & 3,5 \\
Octubre & $6: 00$ & 59,58 & 18,5 & 12 \\
& $7: 00$ & 59,52 & 16 & 6,5 \\
& $8: 00$ & 61,78 & 11 & 8 \\
& $9: 00$ & 61,03 & 11,5 & 14 \\
Noviembre & $6: 00$ & 59,18 & 23 & 8,5 \\
& $7: 00$ & 58,83 & 11,5 & 10 \\
& $8: 00$ & 59,08 & 13 & 11,5 \\
\hline
\end{tabular}

\section{DISCUSIÓN}

El registro de 43 especies de aves canoras en el Jardín Botánico Alejandro von Humboldt representa el $26,87 \%$ de las especies reportadas por Moreno-Palacios, Jeyson-Sanabria, Díaz-Jaramillo, Carantón-Ayala y Parra-Hernández (2007), quienes registraron 160 especies mediante la aplicación de métodos variados como las detecciones visuales, auditivas y el uso de redes de niebla. La notable diferencia entre el total de especies detectadas bioacústicamente y las regis- tradas por medio de los métodos mencionados, radica en que buena parte de las especies inventariadas no presentan amplia actividad vocal, además es frecuente que la detectabilidad de los organismos mediante métodos bioacústicos varíe según la tasa de canto, la época del año, las condiciones climáticas, la hora del día, el tipo de ambiente (Catchpole y Slater, 1995; McShea y Rappole, 1997), la disponibilidad de alimento (Alatalo, Glynn y Lundberg, 1990) y el ruido ambiental. 
La presencia de un alto número de especies de las familias Thraupidae y Tyrannidae corresponde significativamente a algunas zonas abiertas aledañas al jardín botánico, además estas familias presentan un dominante número de especies en la zona de estudio (11 y 26 respectivamente) (Moreno-Palacios et al., 2007), y se caracterizan por presentar alta actividad vocal. También es posible que su notable presencia en el área estudiada se deba a sus preferencias alimenticias, las cuales afectan en menor medida a las especies que son más propensas a las condiciones de la comida urbana siendo típicamente más exitosas las aves omnívoras, frugívoras e insectívoras que las especies carnívoras (Clergeau, Savard, Mennechez y Falardeau, 1998; Lim y Sodhi, 2004).

Las especies con mayor número de detecciones tanto en los meses como en las horas de trabajo, son consideradas como abundantes en la región, pues son comunes en una amplia variedad de hábitats con diferentes grados de intervención antrópica incluyendo la ciudad, y, por tal se categorizan como especies urbanas caracterizadas por presentar un buen grado de adaptación y tolerancia a niveles intensos de ruido vehicular continuo (Slabbekoorn, 2004). Además, según lo postulado por Marzluff (2005), la urbanización por lo general tiene un efecto negativo sobre las especies raras y favorece a otras que se vuelven cada vez más comunes, como las especies generalistas nativas o colonizadoras urbanas (no nativas), afirmación que explica la razón por la cual las especies con IIR superior a 0,5 son las más detectadas en los meses y horas de muestreo sin importar el promedio de ruido vehicular registrado en los mismos (Anexo 1).

Por otro lado, según Catchpole y Slater (1995) y Ralph et al. (1996), para la mayoría de especies las tasas de canto son más altas durante el período entre la primera luz del día (el amanecer y la salida del sol) y disminuye gradualmente hacia el mediodía. En zonas tropicales, los horarios de canto de las diferentes especies pueden variar entre sí, pues estas tienden a comenzar a cantar en diferentes momentos, evidenciándose que las especies que pueden ver mejor a bajas intensidades de luz generalmente inician sus actividades diarias antes del amanecer (Thomas et al., 2002), además, la altura de forrajeo también influye en la hora de inicio del repertorio vocal (Berg, Brumfield y Apanius, 2006). Así, las 6:00 h con su mayor registro de especies (30) y detecciones
(118), y las 9:00 h y sus valores mínimos (23 especies y 58 detecciones), evidencian dicha teoría.

Pater (2001) menciona que existe una dificultad metodológica relacionada con el hecho de suponer el efecto del sonido como una consecuencia aislada de otras variables que expliquen ciertos comportamientos de la fauna silvestre. En muchos ensayos no es claro si las respuestas observadas se deben exclusivamente al sonido o a otros componentes provocadores de disturbio, por tal la discriminación de estos factores tiene especial relevancia a la hora de diseñar estudios de afectación del ruido ambiental con respecto a las diferentes especies (Richardson, Greene, Malme y Thomson, 1995), en este estudio se evidencia que la intensidad del ruido vehicular no incide en el número de especies y detecciones, sino que estas variables se ven principalmente afectadas por la hora de muestreo, sin embargo, no se descarta que en lugares en donde la intensidad del ruido sea menos constante este factor influya sobre la persistencia de las comunidades de aves y su conservación.

Por otra parte, al igual que en estudios desarrollados por Stone (2000), Peris y Pescador (2004) y Llavallol et al. (2012), no se detectaron asociaciones significativas entre la intensidad de ruido y las características de la comunidad de aves incluso cuando la fuente de ruido era cercana, pero la correlación entre el número de detecciones o especies y el nivel sonoro sugieren que el ruido podría incidir sobre estos caracteres. Así, de acuerdo con lo expuesto previamente, el jardín botánico de la Universidad del Tolima se postula como un sitio de interés para el monitoreo y la realización de trabajos que permitan conocer cómo la avifauna que sustenta se ve afectada por los procesos antrópicos desarrollados en el o en sus cercanías (carreteras aledañas), como una forma de contribuir a la preservación y conservación de este fragmento de bosque que actúa como conector biológico entre el área urbana y otras zonas boscosas.

\section{CONCLUSIONES}

No existen diferencias significativas en el número de especies y de detecciones en relación a la intensidad del ruido vehicular, sin embargo, estas variables se ven fuertemente influenciadas por el mes y la hora de muestreo. Además, se evidencia que el notable aumento en el número de detecciones a medida que 
trascurren los meses, se genera principalmente por la transición de la época seca a la lluviosa. Recomendamos realizar más estudios sobre la influencia del ruido en las comunidades de aves tanto en zonas urbanas como semiurbanas y consideramos importante ampliar a escala anual dichos trabajos.

\section{AGRADECIMIENTOS}

Jessica Nathalia Sánchez-Guzmán agradece al Grupo de Investigación en Zoología por la logística durante los meses de pasantía del Programa de Biología de la Universidad del Tolima.

\section{REFERENCIAS}

Alatalo, R. V., Glynn, C. \& Lundberg, A. (1990). Singing rate and female attraction in the Pied flycatcher: An experiment. Animal Behaviour, 39(3), 601-603.

Arévalo, J. E. \& Newhard, K. (2011). Traffic noise affects forest bird species in a protected tropical forest. Revista de Biología Tropical, 59(2), 969-980.

Audacity Team (2013). Audacity ${ }^{\circledR}$ Version 2.0.5. Audio editor and recorder. Disponible en: http:// audacity.sourceforge.net/

Bee, M. A. \& Swanson, E. M. (2007). Auditory masking of anuran advertisement calls by road traffic noise. Animal Behaviour, 74(6), 1765-1776.

Berg, K. S., Brumfield, R. T. \& Apanius, V. (2006). Phylogenetic and ecological determinants of the Neotropical dawn chorus. Proceedings of the Royal Society B: Biological Sciences, 273, 999-1005.

Brattstrom, B. H. \& Bondello, M. C. (1983). Effects of offroad vehicle noise on desert vertebrates. En Webb R.H. \& Wilshore H.G. (eds.). Environmental effects of off-road vehicles. Impacts and management in arid regions. (pp. 167-206). New York: SpringerVerlag.

Bucher, E. H. \& Herrera, G. (1981). Comunidades de aves acuáticas de la laguna Mar Chiquita (Córdoba, Argentina). Ecosur, 8(15), 91-120.

Catchpole, K. C. \& Slater, P. J. B. (1995). Bird song: Biological themes and variations. (pp. 113-138). New York: Cambridge University Press. 2 ed.
Clergeau, P., Savard, J-P. L., Mennechez, G. \& Falardeau, G. (1998). Bird abundance and diversity along an urban-rural gradient: a comparative study between two cities on different continents. Condor, 100(3), 413-425.

Di Rienzo, J. A., Casanoves, F., Balzarini, M. G., González, L., Tablada, M. \& Robledo, C. W. InfoStat versión 2013. Grupo InfoStat, FCA, Universidad Nacional de Córdoba, Argentina. Disponible en: http://www.infostat.com.ar

Fahrig, L., Pedla, J. H., Pope, S. E., Taylor, P. D. \& Wegner, J. F. (1995). Effect of road traffic on amphibian density. Biological Conservation, 73(3), 177-182.

Fernández-Juricic, E. (2000). Bird community composition patterns in urban parks of Madrid: the role of age, size, and isolation. Ecological Research, 15(4), 373-383.

Francis, C. D., Ortega, C. P. \& Cruz, A. (2009). Noise pollution changes avian communities and species interactions. Current Biology, 19(16), 1415-1419.

Francis, C. D., Ortega, C. P. \& Cruz, A. (2011). Vocal frequency change reflects different responses to anthropogenic noise in two suboscine tyrant flycatchers. Proceedings of the Royal Society B: Biological Sciences, 278(1714), 2025-2031.

Gatto, A., Quintana, F., Yorio, P. \& Lisnizer, N. (2005). Abundancia y diversidad de aves acuáticas en un humedal marino del golfo San Jorge, Argentina. Hornero, 20(2), 141-152.

Goodwin, S. E. \& Shriver, W. G. (2013). Effects of traffic noise on occupancy patterns of forest birds. Conservation Biology, 25(2), 406-411.

Goosem, M. (2002). Effects of tropical rainforest roads on small mammals: fragmentation, edge effects and traffic disturbance. Wildlife Research, 29(3), 277-289.

Llavallol, C., Cellini, J. M. \& Gutiérrez, M. (2012). Impacto del ruido sobre la riqueza y abundancia de las aves, en el bosque de arrayanes (Luma apiculata (DC) Burret) en península de Quetrihue, Argentina. UNLaR Ciencia, 1(1), 20-26. 
Lengagne, T. (2008). Traffic noise affects communication behaviour in a breeding anuran, Hyla arborea. Biological Conservation, 141(8), 2023-2031.

Lim, H. C. \& Sodhi, N. S. (2004). Responses of avian guilds to urbanization in a tropical city. Landscape and Urban Planning, 66(4), 199-215.

Ospina-López, L. A. \& Reinoso-Flórez, G. (2009). Mariposas diurnas (Lepidoptera: Papilionoidea y Hesperioidea) del Jardín Botánico Alejandro von Humboldt de la Universidad del Tolima (Ibagué Colombia). Tumbaga, 1(4), 135-148.

Lukanov, S., Simeonovska-Nikolova, D. \& Tzankov, N. (2014). Effects of traffic noise on the locomotion activity and vocalization of the Marsh Frog, Pelophylax ridibundus. North-Western Journal of Zoology, 10(2), 359-364.

Mace, R. D., Waller, J. S., Manley, T. L., Lyon, L. J. \& Zuuring, H. (1996). Relationships among grizzly bears, roads and habitat in the Swan Mountains, Montana. Journal of Applied Ecology, 33(6), 13951404.

Marzluff, J. M. (2005). Island biogeography for an urbanizing world: how extinction and colonization may determine biological diversity in humandominated landscapes. Urban Ecosystems, 8(2), 157-177.

McGregor, R. L., Bender, D. J. \& Fahrig, L. (2008). Do small mammals avoid roads because of the traffic? Journal of Applied Ecology, 45(1), 117-123.

McShea, W. J. \& Rappole, J. H. (1997). Variable song rates in three species of passerines and implications for estimating bird populations. Journal of Field Ornithology, 68(3), 367-375.

Mendes, S., Colino-Rabanal, V. J. \& Peris, S. J. (2011). Diferencias en el canto de la ratona común (Troglodytes musculus) en ambientes con distintos niveles de influencia humana. Hornero, 26(2), 8593.

Mendoza, A. M. \& Arce-Plata, M. I. (2012). Aproximación al impacto de la perturbación urbana en las vocalizaciones de Pitangus sulphuratus (Tyrannidae) en Santiago de Cali, Valle del Cauca
(Colombia). Revista de Ciencias Universidad del Valle, 16, 19-29.

Moreno-Palacios, M., Jeyson-Sanabria, J., DíazJaramillo, C., Carantón-Ayala, D. \& ParraHernández, R. (2007). Caracterización de la avifauna del Jardín Botánico Alejandro von Humboldt Universidad del Tolima. Inf. téc. Jardín Botánico AvH- Universidad del Tolima. Ibagué.

Pacheco-Vargas, G. \& Losada-Prado, S. (2015). Efecto del ruido del tráfico vehicular en cantos de Hylophilus flavipes y Cyclarhis gujanensis. Revista Ciencia en Desarrollo, 6(2), 177-183.

Pater, L. (2001). Defining auditory thresholds for animal species. En Baker M. \& Belliveau G. (ed.). Proceedings of the effects of noise on wildlife conference. (pp. 22-25). Happy Valley-Goose Bay, Labrador: Institute for environmental monitoring and research.

Parris, K. M., Velik-Lord, M. \& North, J. M. A. (2009). Frogs call at a higher pitch in traffic noise. Ecology and Society, 14(1), 25.

Perepelizin, P.V. \& Faggi, A. M. (2009). Diversidad de aves en tres barrios de la ciudad de Buenos Aires, Argentina. Multequina, 18(2), 71-85.

Peris, S. J. \& Pescador, M. (2004). Effects of traffic noise on passerine populations in Mediterranean wooded pastures. Applied Acoustics, 65(4), 357366.

Pisanty, I., Mazari, M., Ezcurra, E., et al. (2009). El reto de la conservación de la biodiversidad en zonas urbanas y periurbanas, en capital natural de México. En Dirzo, R., González, R. \& March, I.J. (ed.). Estado de conservación y tendencias de cambio. (pp. 719-759). México: Conabio.

Ralph, C. J., Geupel, G. R., Pyle, P., Martin, T. E., DeSante, D. F. \& Borja, M. (1996). Manual de métodos de campo para el monitoreo de aves terrestres (p. 46). Gen. Tech. Rep. PSW-GTR-159. Albany, CA: Pacific Southwest Research Station, Forest Service, U.S. Department of Agriculture.

Richardson, W. J., Greene, C. R., Malme, C. I. \& Thomson, D. H. (1995). Marine mammals and noise (p. 576). San Diego CA: Academy press Inc. 
Rost, G. R. \& Bailey, J. A. (1979). Distribution of mule deer and elk in relation to roads. Journal of Wildlife Management, 43(3), 634-641.

Ruiz, A., Rubines, J. \& Lahoz, E. (2006). Efecto de la contaminación acústica sobre las poblaciones de vertebrados forestales en Álava. Asociación medioambiental ATTHIS. Disponible en: http:// www.ingurumena.ejgv.euskadi.eus/r49- 3074/ es/contenidos/informe_estudio/contaminacion_ acustica_vertebr/es_doc/adjuntos/memoria.pdf

Slabbekoorn, H. \& Peet, M. (2003). Ecology: Birds sing at a higher pitch in urban noise. Nature, 424(6946), 267.

Slabbekoorn, W. H. (2004). Singing in the wild: The science of birdsong. En Marler, P. \& Slabbekoorn W. H. (eds.). Nature's music. (pp. 178-205). San Diego, California: Elservier Academic Press.

Slabbekoorn, H. \& den Boer-Visser, A. (2006) Cities change the songs of birds. Current Biology, 16(23), 2326-2331.

Slabbekoorn, H., Yang, X. J. \& Halfwerk, W. (2012). Birds and Anthropogenic Noise: Singing Higher May Matter (A Comment on Nemeth and Brumm, "Birds and Anthropogenic Noise: Are Urban Songs Adaptive?"). The American Naturalist, 180(1), 142-145.

Stone, E. (2000). Separating the noise from the noise: a finding in support of the 'niche hypothesis', that birds are influenced by human-induced noise in natural habitats. Anthrozoos, 13(4), 225-231.

Sun, J. W. C. \& Narins, P. (2005). Antropogenic sounds differentially affect amphibians call rate. Biological Conservation, 121(3), 419-427.

Thomas, R. J., Szekely, T., et-al. (2002). Eye size in birds and the timing of song at dawn. Proceedings of the Royal Society B: Biological Sciences, 269(1493), 831-837.

Wollerman, L. \& Wiley, R. H. (2002). Possibilities for error during communication by Neotropical frogs in a complex acoustic environment. Behavioral Ecology and Sociobiology, 52(6), 465-473. 
Anexo 1. Frecuencia de ocurrencia absoluta y relativa (FOR), índice de importancia relativa (IIR) y abundancia relativa de las especies de aves registradas mediante herramientas bioacústicas en el Jardín Botánico Alejandro von Humboldt de la Universidad del Tolima, Ibagué (Colombia) (SACC Classification version 26 May 2014).

\begin{tabular}{|c|c|c|c|c|c|c|}
\hline \multicolumn{3}{|c|}{ Datos taxonómicos } & \multicolumn{2}{|c|}{ Frecuencia de ocurrencia } & \multirow{2}{*}{ IIR } & \multirow{2}{*}{$\begin{array}{l}\text { Abundancia } \\
\text { relativa (\%) }\end{array}$} \\
\hline ORDEN & FAMILIA & ESPECIES & Absoluta & Relativa (\%) & & \\
\hline Accipitriformes & Accipitridae & Rupornis magnirostris & 4 & 57,14 & 2,40 & 1,2 \\
\hline Gruiformes & Rallidae & Aramides cajaneus & 1 & 14,29 & 0,05 & 0,3 \\
\hline Charadriiformes & Charadriidae & Vanellus chilensis & $2^{*}$ & 28,57 & 0,33 & 0,6 \\
\hline Columbiformes & Columbidae & Columbina talpacoti & 2 & 28,57 & 0,54 & 0,6 \\
\hline Caprimulgiformes & Caprimulgidae & Nyctidromus albicollis & 1 & 14,29 & 0,05 & 0,3 \\
\hline Apodiformes & Trochilidae & Amazilia tzacalt & $2^{*}$ & 28,57 & 0,16 & 0,6 \\
\hline Coraciiformes & Momotidae & Momotus aequatorialis & 1 & 14,29 & 0,05 & 0,3 \\
\hline Galbuliformes & Bucconidae & Malacoptila mystacalis & 1 & 14,29 & 0,33 & 0,3 \\
\hline Piciformes & Picidae & Melanerpes rubricapillus & $5^{*}$ & 71,43 & 1,31 & 1,5 \\
\hline Psittaciformes & Psittacidae & Brotogeris jugularis & 6 & 85,71 & 10,07 & 1,8 \\
\hline Passeriformes & Thamnophilidae & Thamnophilus doliatus & 4 & 57,14 & 0,65 & 1,2 \\
\hline Passeriformes & Thamnophilidae & Myrmeciza longipes & $2^{*}$ & 28,57 & 0,16 & 0,6 \\
\hline Passeriformes & Tyrannidae & Elaenia flavogaster & 1 & 14,29 & 0,05 & 0,3 \\
\hline Passeriformes & Tyrannidae & Elaenia chiriquensis & 1 & 14,29 & 0,05 & 0,3 \\
\hline Passeriformes & Tyrannidae & Phaeomyias murina** & $1^{*}$ & 14,29 & 0,03 & 0,3 \\
\hline Passeriformes & Tyrannidae & Todirostrum cinereum ${ }^{* *}$ & $1^{*}$ & 14,29 & 0,05 & 0,3 \\
\hline Passeriformes & Tyrannidae & Myiozetetes similis & $3^{*}$ & 42,86 & 0,41 & 0,9 \\
\hline Passeriformes & Tyrannidae & Pitangus sulphuratus & $7^{*}$ & 100 & 4,49 & 2,1 \\
\hline Passeriformes & Tyrannidae & Megarynchus pitangua & 3 & 42,86 & 0,82 & 0,9 \\
\hline Passeriformes & Vireonidae & Cyclarhis gujanensis & 6 & 85,71 & 5,44 & 1,8 \\
\hline Passeriformes & Vireonidae & Hylophilus flavipes & $6^{*}$ & 85,71 & 7,62 & 1,8 \\
\hline Passeriformes & Troglodytidae & Troglodytes aedon & $7^{*}$ & 100 & 11,08 & 2,1 \\
\hline Passeriformes & Troglodytidae & Pheugopedius fasciatoventri & 2 & 28,57 & 0,54 & 0,6 \\
\hline Passeriformes & Troglodytidae & Henicorhina leucosticta** & $1^{*}$ & 14,29 & 0,03 & 0,3 \\
\hline Passeriformes & Turdidae & Turdus leucomelas & 2 & 28,57 & 0,16 & 0,6 \\
\hline Passeriformes & Turdidae & Turdus ignobilis & $6^{*}$ & 85,71 & 3,27 & 1,8 \\
\hline Passeriformes & Thraupidae & Eucometis penicillata & 1 & 14,29 & 0,05 & 0,3 \\
\hline Passeriformes & Thraupidae & Tachyphonus /uctuosus & 1 & 14,29 & 0,33 & 0,3 \\
\hline Passeriformes & Thraupidae & Ramphocelus dimidiatus & $7^{*}$ & 100 & 5,69 & 2,1 \\
\hline Passeriformes & Thraupidae & Thraupis episcopus & $7^{*}$ & 100 & 5,99 & 2,1 \\
\hline Passeriformes & Thraupidae & Thraupis palmarum ${ }^{* *}$ & $1^{*}$ & 14,29 & 0,03 & 0,3 \\
\hline Passeriformes & Thraupidae & Tangara vitriolina & 3 & 42,86 & 0,4 & 0,9 \\
\hline Passeriformes & Thraupidae & Tangara cyanicollis & 3 & 42,86 & 0,68 & 0,9 \\
\hline Passeriformes & Thraupidae & Tangara girola & 1 & 14,29 & 0,05 & 0,3 \\
\hline Passeriformes & Thraupidae & Dacnis cayana & 1 & 14,29 & 0,05 & 0,3 \\
\hline Passeriformes & Thraupidae & Sicalis flaveola & 3 & 42,86 & 0,65 & 0,9 \\
\hline
\end{tabular}




\begin{tabular}{|c|c|c|c|c|c|c|}
\hline \multicolumn{3}{|c|}{ Datos taxonómicos } & \multicolumn{2}{|c|}{ Frecuencia de ocurrencia } & \multirow{2}{*}{ IIR } & \multirow{2}{*}{$\begin{array}{l}\text { Abundancia } \\
\text { relativa (\%) }\end{array}$} \\
\hline ORDEN & FAMILIA & ESPECIES & Absoluta & Relativa (\%) & & \\
\hline Passeriformes & Thraupidae & Sporophila minuta & 1 & 14,29 & 0,05 & 0,3 \\
\hline Passeriformes & Thraupidae & Coereba flaveola & 1 & 14,29 & 0,05 & 0,3 \\
\hline Passeriformes & Thraupidae & Tiaris bicolor & $4^{*}$ & 57,14 & 0,82 & 1,2 \\
\hline Passeriformes & Incertae Sedis & Saltator striatipectus & $7^{*}$ & 100 & 7,49 & 2,1 \\
\hline Passeriformes & Parulidae & Myiothlypis fulvicauda & $7^{*}$ & 100 & 9,28 & 2,1 \\
\hline Passeriformes & Parulidae & Basilleuterus rufifrons & 1 & 14,29 & 0,05 & 0,3 \\
\hline Passeriformes & Fringillidae & Euphonia laniirostris & $3^{*}$ & 42,86 & 0,68 & 0,9 \\
\hline
\end{tabular}

*Frecuencias de ocurrencia absolutas que incluyen detecciones de algún individuo en el mes de agosto.

**Especies registradas en agosto y que no fueron detectadas en los demás meses. 